\title{
EDITORIAL
}

\section{OPERAÇÃO DE JATENE: $1975-1987$}

\author{
Prof. Dr. Adib D. Jatene \\ Editor
}

Em maio de 1985, JATENE et alii ${ }^{4}$ realizaram a primeira correção da transposição das grandes artérias no plano arterial e a denominaram correção anatômica. Em outubro do mesmo ano, esta operação foi apresentada como comentário em mesa-redonda do II Simpósio do Henry Ford Hospital ${ }^{3}$. O fato deste simpósio reunir cirurgiōes do mundo inteiro e a circunstância de este tipo de correção estar na mente de todos. como algo irrealisável, causou um grande impacto.

A técnica passou a ser utilizada em vários Serviços ${ }^{2}$ 8. 12 e a ser chamada "Operação de Jatene". Sua indicação eletiva era para os casos de transposição com comunicação interventricular e/ou persistência de canal arterial. Também pacientes com estenose pulmonar que permitisse sua eliminação completa com boa função valvar passaram a ser indicados. Quanto à transposição com septo íntegro, todos os grupos se mantiveram indicando a correção no plano atrial. Apenas YACOUB et alii ${ }^{13}$ imaginaram preparar o ventrículo esquerdo com a cerclagem da artéria pulmonar, com ou sem desvio aorto-pulmonar. Inicialmente, faziam o desvio proximal à cerclagem. Em seguida, deixaram de fazê-lo e empregavam apenas a cerclagem e, ao final, o desvio aorto-pulmonar passou a ser realizado distalmente à cerclagem.

As discussões sobre qual o método a aplicar, se no plano atrial, com as operações de MUSTARD ${ }^{9}$ e de SENNING ${ }^{11}$, ou no plano arterial, com a operação de JATENE $^{5}$, com ou sem a modificação de LECOMPTE et alii ${ }^{7}$, tornaram-se assunto obrigatório, nos congressos de Cardiologia Pediátrica.

Permanecia, porém, a indicação, principalmente para os casos complexos, que representam menos de $20 \%$. A indicação em casos simples, ou seja, com septo íntegro, exigia a preparação prévia proposta por Yacoub. A não ser que se operassem os pacientes antes que houvesse adelgaçamento das paredes do ventrículo esquerdo. Isto queria dizer que a cirurgia deveria ser realizada no período neonatal.

Depois de CASTAÑEDA et alii ${ }^{1} \mathrm{e}$, mais recentemente, QUAEGEBEUR ei alii ${ }^{10}$ apresentaram suas experiências no período neonatal é que o conceito realmente começou a mudar.

Atualmente, um grupo multicêntrico, liderado por KIRKLIN \& BLACKSTONE ${ }^{6}$, ocupa-se da avaliação da técnica mais adequada para correção definitiva da transposição das grandes artérias.

Após 12 anos e inúmeras discussões, parece que a operação de Jatene, como foi proposta, ou com modificações, vai ganhando espaço e tudo leva a crer que substituirá as outras técnicas, em todos os Serviços preparados para cirurgia eletiva em neonatos.

Mesmo nos doentes com septo ventricular íntegro que ultrapassaram a fase neonatal, a preparação do ventrículo esquerdo, como proposta por Yacoub, permite a realização da operação de Jatene.

O processo de validação desta técnica, até sua aceitação definitiva, como ocorre hoje, demonstra, claramente, o cuidado dos cirurgiōes na adoção de procedimentos novos e a certeza de que, ao contrário das aparências, que levam a considerar os cirurgiōes como agressivos, eles são extremamente prudentes e conservadores, só mudando de atitude quando a análise cuidadosa dos resultados justificar esta mudança. 


\section{REFERÊNCIAS BIBLIOGRÁFICAS}

1 CASTAÑEDA, A. R.; NORWOOD. W. J.; JONAS, R. A.; COLON, S. D.; SANDERS, S. P.; LANG, P. - Transposition of the great arteries and intact ventricular septum: anatomical repair in the neonate. Ann. Thorac. Cardiovasc. Surg., 38 (5): 438-443, 1984.

2 HARINK, E.; VAN MILL, G. J.; ROSS, D. - Anatomical correction of transposition of great arteries with persistent ductus arteriosus: one year after operation. Br. Heart. J., 43 (1): 95-98, 1980.

3 JATENE, A. D. - Discussion at the round table on Surgery for complex congenital anomalies. In: Henry Ford Hospital International Symposium on Cardiac Surgery. 2., 1975. Detroit, USA, Appleton-Century-Croft, 1977. p. 335-338.

4 JATENE, A. D.; FONTES, V. F.; PAULISTA, P. P.; SOUZA, L. C. B.; NEGER, F.: GALANTIER, M.; SOUSA, J. E. M. R. - Successful anatomic correction of transposition of the great vessels: a preliminary report. Arq. Bras. Cardiol., 28 (4): 461-464, 1975.

5 JATENE, A. D.; FONTES, V. F.; PAULISTA, P. P.: SOUZA, L. C. B.; NEGER, F.; GALANTIER, M.; SOUSA, J. E. M. R. - Anatomic correction of transposition of the great vessels. J. Thorac. Cardiovasc. Surg., 72 (3): 364-370, 1976.

6 KIRKLIN, J. W. \& BLACKSTONE, E. H. - An ongoing study of the management of patients with transposition to the great arterires. Congenital Heart Surgeons Society, Birmingham, USA, 1986, $108 \mathrm{p}$.
7 LECOMPTE, Y.; ZANNINI, L.; HARAN, E.; JARREAU, M. M.; BEX, J. P.; TRAN VIET TU; NEVEAUX, J. Y. Anatomic correction of transposition of the great arteries new technique without use of a prosthetic conduit. J. Thorac. Cardiovasc. Surg., 82 (4): 629-631, 1981.

8 MAJOR Jr., W. K.; MATSUDA, H.; SUBRAMANIAN, S. - Failure of the Jatene procedure in a patient with d-transposition and intact ventricular septum. $A \cap n$. Thorac. Surg., 22 (1): 386-388, 1976.

9 MUSTARD, W. T.; CHUTE, A. L.; KEITH, J. D.; SIREK, A.; ROWE, R. D.; VLAD, R. - A surgical approach to transposition of the great vessels with extracorporeal circuit. Surgery, 36 (1): 39-51, 1954.

10 QUAEGEBEUR, J. M.: ROHMER, J.; OTTENKAMP, J.; BUIS, T.: KIRKLIN. J. W.: BLACKSTONE, E.; BROM. A. G. - The arterial switch operation: an eight-year experience. J. Thorac. Cardiovasc. Surg., 92 (3): 361-384, 1986.

11 SENNING, A - Surgical correction of transposition of the great vessels. Surgery, 45 (6): 966-980, 1959.

12 STARR, A. - Discussion of Jatene AD, Fontes VF, Paulista PP. Souza LCB, Neger F, Galantier M, Souza JEMR: Anatomic correction of transposition of the great verssels. J. Thorac. Cardiovasc. Surg., 72 (3): 364-370, 1976.

13 YACOUB, M. H.; RADLEY-SMITH, R.; MacLAURIN, K. - Two-stage operation for anatomical correction of transposition of the great arteries with intact interventricular septum. Lancet. 1 (8025): 1275-1278, 1977. 